\title{
Affective Brain-Computer Interfacing and Methods for Affective State Detection
}

\author{
Ian Daly* \\ Brain-Computer Interfacing and Neural Engineering Laboratory, \\ School of Computer Science and Electronic Engineering, \\ University of Essex, Colchester, CO4 3SQ, UK
}

June 6, 2017

\begin{abstract}
Affective brain-computer interfaces (aBCIs) provide a method for individuals to interact with a computer via their emotions and without needing to move.

This chapter will provide an introduction to the concept of aBCIs and their uses in applications such as music therapy and affective computing. We will first review the concept of aBCIs before going on to provide a literature review of the current state-of-the-art research in affective state detection methods and their uses in aBCI. Finally, we will describe a case study; an affective brain-computer music interface (aBCMI) and its potential for use in music therapy.

Emerging and established trends in aBCI, such as the use of pre-frontal asymmetry measures of affective states, are identified. Additionally, a set of recommendations are provided for researchers seeking to work in the field of aBCI.
\end{abstract}

\section{Introduction}

Brain-computer interfaces (BCIs) seek to provide a channel for communication and control of a computer system that does not rely on any movement and instead uses signals recorded directly from the users brain to achieve interaction with a computer [1]. Affective BCIs (aBCIs) seek to detect a users affective state and use that information to interact with the BCI $[2,3,4]$. aBCIs may seek to detect a users affective state either directly from their brain or via the use of a hybrid approach that combines both brain activity and measures of other

*email: i.daly@essex.ac.uk; 
physiological processes. aBCIs have a wide range of potential applications, including aiding with affective computing, entertainment, and therapy.

This chapter will first introduce affective states and how they may be categorized It will then go on to discuss methods for individuals to report their affective states before introducing methods for affective state detection based on recordings of neurological and physiological activity. A literature review of the current-state-of-the-art in aBCIs will then be presented before a final case study, which describes a recent development in aBCIs with potential applications for music therapy. Finally, a set of guidelines will be provided to aid with the development of aBCIs.

\section{Affective states}

An affective state is defined as a psychological construct which seeks to categorize the experience of feeling an emotion [5]. Affect is often measured along either two or three different axes, of which the most commonly used are 'valance', which refers to how pleasant or unpleasant an experience is, 'arousal', which refers to how exciting or unexciting an experience is, and 'tension', which refers to how much or little tension is felt during an experience [6].

Taken together these different axes may be used to model an affective space that seeks to encompass the range of human emotion. Several different models of the affective space exist, using different combinations of axes to attempt to allow all possible affective states to be collated within the model and consequently measured and analytically evaluated.

One of the more popular and frequently used such models is the valancearousal circumplex model [7]. This model uses two axes, valance and arousal, to map the affective space onto a two dimensional plane. A range of different discrete affective states are then mapped to this affective space by identifying their locations on the valance and arousal axes. For example, 'joy' may be mapped to the upper right hand corner of the space because it corresponds to a high valance (very pleasant) state and a high arousal (very excited) state. The valence-arousal circumplex model is illustrated in figure 1.

A range of other models of the affective space have also been proposed. These include the Schimack and Grob 3-dimensional model, which describes the affective space via the three dimensions of valence, energy-arousal, and tensionarousal [8], and the Geneva Emotional Music Scale (GEMS), which seeks to describe the affective space via a set of discrete state labels that were selected for their relevance to the set of emotions commonly considered to be conveyable via music [9].

GEMS differs from many other models of affect by utilizing labeled categories to describe an affective state in place of a continuous space. For example, the GEMS-45 system contains 45 discrete labels, grouped under 9 categories, that describe affective states such as 'wonder' or 'joyful activation'. Different combinations of labels or individuals labels may be used to describe a single affective state. 


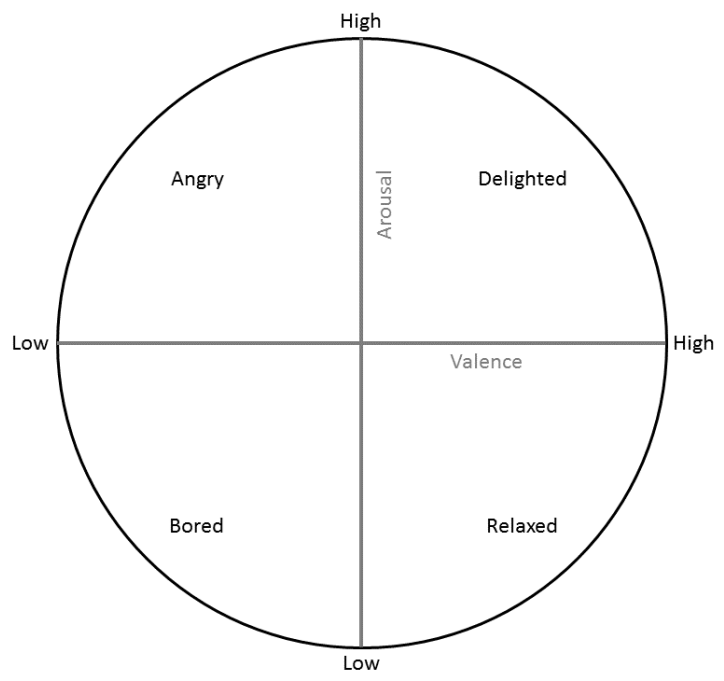

Figure 1: An example of the valence-arousal circumplex that may be used to model affective states along the two axes of valence and arousal.

Although affect may be described by discrete states or labels the actual processes involved in the experience of affect are widely understood to be multimodal and involve multiple different neurological and physiological processes. Specifically, when you feel an emotion this is the result of a complex interaction of multiple neurological and physiological processes acting at different time scales and influencing one another to together result in the embodied feeling of an affect [10].

For example, your heart rate changes with differing levels of arousal [11, 12, 13], while skin conductivity (measured by galvanic skin response (GSR)) has been reported to change with changing levels of arousal, valence, and tension [14, $15,16]$. Additionally, respiration rate has been reported to change in response to changing levels of both valence and arousal $[17,18]$. Thus, an individual's affective state may be understood to be a combination of their brain and body responding to changes in their situation (either triggered by external stimuli or internal processes such as memory recall of emotive events) $[5,19]$.

With regards to external stimuli, affect can either be felt or perceived [20]. Perceived affect is an individuals understanding of the affective state a stimuli is attempting to convey, while felt affect refers to the actual affective state experienced by the individual when exposed to the stimuli. An illustrative example of this is the enjoyment of 'sad' music. A listener may perceive that a piece of music is attempting to convey sadness and yet derive considerable pleasure from listening to it [20]. 


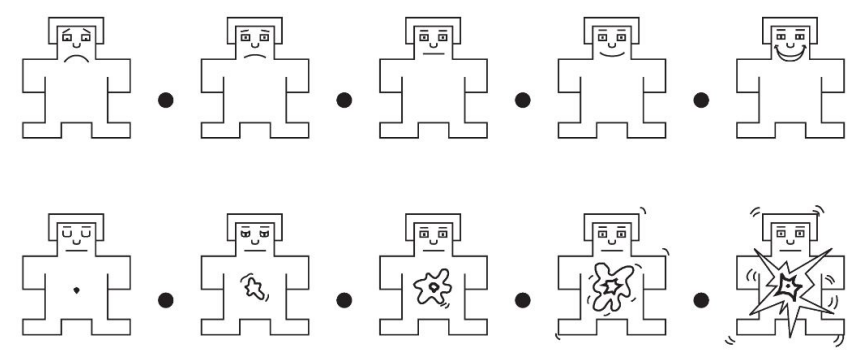

Figure 2: Self assessment manikin (SAM) method of affective state reporting.

\section{$3 \quad$ Affective state reporting}

When studying affect (e.g. the affective response of an individual or group to stimuli) it is often necessary to provide some mechanism for the individual to report their current felt or perceived affective state. Consequently, a variety of tools have been developed to allow the recording of users reports of their affective states.

These tools are useful to consider in the development of aBCIs as, when developing tools and component parts of an aBCI it is often necessary to have a ground truth measure of an individuals current affective state. For example, when developing a classifier to detect a user's current felt affective state in an aBCI user it may often be necessary to train the classifier on some ground truth measure.

Affective state reporting tools may be described as either discrete or continuous tools. Discrete tools are used for recording single discrete snapshots of a users affective state at one particular moment in time or over one discrete time window. By way of contrast, continuous affect reporting tools allow users to provide a moment-by-moment report of their affective state as it changes over time.

\subsection{Discrete methods}

There are a variety of discrete affective state reporting tools available. Two of the most widely used in psychological studies of affect are the self assessment manikin (SAM) and the Geneva Emotional Music Scale (GEMS).

The SAM provides a set of figures depicting different positions within the valence arousal space [21]. These are illustrated in figure 2. Participants are asked to select different positions on the space that match the affective state they wish to report.

The SAM is able to provide a discrete snapshot of affect at a single moment in time. Furthermore, it does so via the use of pictographic representations of affect, which may be less susceptible to inter-personal differences in understanding of linguistics descriptors of affect in different languages [22]. Thus, there is 


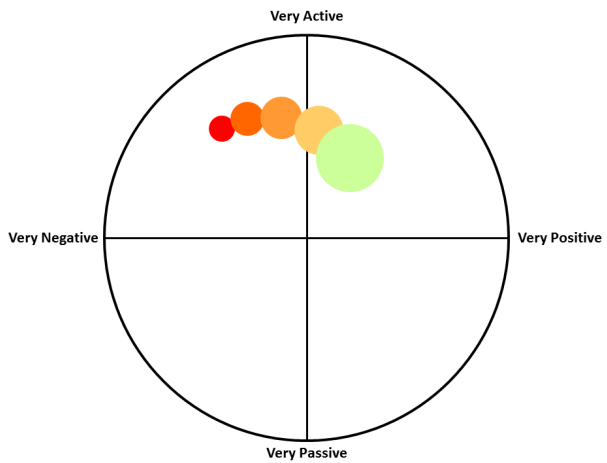

Figure 3: The FEELTRACE interface for continuous reporting of affective states. The user control the position of the cursor via a mouse or joystick and positions it at a location in the valence-arousal circumplex that best matches how they are feeling at that moment in time.

no need to translate the SAM scale into different languages. However, the use of SAM does require participants to understand the concepts of the valence and arousal scales that are employed before they can use it effectively.

By way of contrast, the GEMS scale is comprised of a set of labels describing different regions of the affective space $[9,23]$. Individuals are asked to select specific labels that correspond to the regions matching how they wish to report their affective state. For example, they may be asked to select labels corresponding to feelings of nostalgia after hearing a piece of music. This allows for more immediate understanding by participants in an experiment, but requires the GEMS scale to be translated into a language the participant is able to understand.

\subsection{Continuous methods}

Several tools that are available for continuous reporting of affective states, some examples include FEELTRACE and GTRACE.

The FEELTRACE tool allows participants to report an affective state on a continuous basis [24]. The tool comprises a two dimensional on screen interface with valence mapped to the horizontal axis and arousal mapped to the vertical axis. Participants can report their current affective state by moving a mouse, joystick, or other input system to position a cursor at a position on the space that best matches the affective state they wish to report.

An example of the FEELTRACE reporting tool is illustrated in figure 3. The tool allows individuals to report their affective state via a relatively simple representation of the valence-arousal space, but can cause confusion in some less computer literate individuals.

To resolve this, a new version of FEELTRACE has recently been developed known as GTRACE [25]. GTRACE provides two one-dimensional reporting 
systems to allow individuals to report an affective state on either the valence or arousal scales independently By separating the valence and arousal axes it has also been reported that GTRACE can act to remove some potential sources of confusion that are related to the simultaneous reporting of different axis in the affective space [25].

\section{Affective state detection}

A key component of aBCI systems is the ability to identify a users current affective state [4]. By detecting a users affective state it is possible for BCIs to respond to a users changing emotions. However, this provides a potentially very personal insight into how an individual is feeling and it is important to consider related privacy and ethical issues (see (?Chapter link: privacy and ethics in brain-computer interface research?)). To do this a variety of different approaches have been developed. These can be based on either just neurophysiological activity or a combination of neurophysiological activity and other physiological activity (a hybrid BCI approach [26, 27]).

BCIs (and by extension aBCIs) can be developed using a variety of different neurological signal types such as near infrared spectroscopy (fNIRS), magnetic encephlography (MEG), or functional magnetic resonance imaging (fMRI) [28]. However, one of the most frequently used methods for measuring neurological activity used in BCI is the electroencephalogram (EEG) [29]. Consequently, the majority of affective state detection methods developed for aBCIs have been developed for EEG, with relatively fewer affective state detection methods for aBCI developed for use with other modalities, such as fMRI [30].

When considering only the EEG as the signal from which to detect affective states the affective state detection methods that have been developed for aBCI systems and other affective computing applications use a range of different types of features. These features may be grouped into the following categories.

1. Band-power based features

2. Asymmetry features

3. Network features

4. Event-related potentials

The first three feature types are not phase locked to the stimulus and can be used on a continuous basis, while event-related potentials (ERPs) are phase locked to a particular stimuli presentation time. Thus, the first three feature types can be used with either synchronous or asynchronous BCI, while ERPs can only be used with synchronous (cue-based) BCIs [31]. These different types of features and their use in affective state detection are detailed in Table 1, along with references to studies that employ these features for identifying an individuals affective state. 
Table 1: Different types of features that may be used in identifying affective states in aBCIs.

\begin{tabular}{|c|c|c|c|}
\hline Feature type & Description & Measured by & References \\
\hline $\begin{array}{l}\text { Band power } \\
\text { features }\end{array}$ & $\begin{array}{l}\text { Relative or absolute changes } \\
\text { in the magnitude of ongoing } \\
\text { non-phase locked oscillatory } \\
\text { activity within one or more } \\
\text { frequency bands over specific } \\
\text { cortical regions. }\end{array}$ & $\begin{array}{l}\text { FFT, } \\
\text { Wavelets } \\
\text { etc. }\end{array}$ & 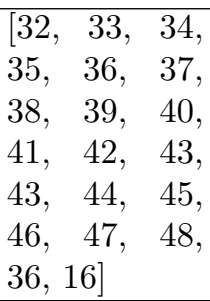 \\
\hline $\begin{array}{l}\text { Asymmetry } \\
\text { features }\end{array}$ & $\begin{array}{l}\text { Relative differences in neural } \\
\text { activity between different re- } \\
\text { gions of the cortex, most typ- } \\
\text { ically between the left and } \\
\text { right hemispheres. }\end{array}$ & $\begin{array}{l}\text { FFT, } \\
\text { Wavelets, } \\
\text { Absolute } \\
\text { or relative } \\
\text { magnitude } \\
\text { differences } \\
\text { etc. }\end{array}$ & $\begin{array}{l}{\left[\begin{array}{lll}49, & 34, & 35, \\
37, & 50, & 51, \\
52, & 42, & 53, \\
47, & 54, & 55]\end{array}\right.}\end{array}$ \\
\hline $\begin{array}{l}\text { Network fea- } \\
\text { tures }\end{array}$ & $\begin{array}{l}\text { Connectivity or associativity } \\
\text { between different regions of } \\
\text { the cortex. }\end{array}$ & $\begin{array}{l}\text { PLV, Coher- } \\
\text { ence etc. }\end{array}$ & $\begin{array}{lll}{[56,} & 57, & 55, \\
58] & & \end{array}$ \\
\hline $\begin{array}{l}\text { Event- } \\
\text { related } \\
\text { potentials }\end{array}$ & $\begin{array}{l}\text { Phase locked changes in EEG } \\
\text { amplitude. }\end{array}$ & $\begin{array}{l}\text { Amplitude } \\
\text { averaging }\end{array}$ & $\begin{array}{lll}{[59,} & 60, & 61, \\
62] & & \end{array}$ \\
\hline
\end{tabular}


Band-power based features measure changes in ongoing oscillatory activity within the EEG. They are not phase locked to a stimulus and, consequently, may be used to measure ongoing changes in affective states in response to continuously changing stimuli. They are most frequently measured via a fast Fourier transform (FFT) or a Wavelet decomposition of the EEG signals. The specific frequency and spatial regions over which these band-powers may be measured differ from study to study. However, there are common regions over which they are most frequently measured.

Table 2 lists the spatial and frequency regions that band-power features are measured over and lists the number of participants over which these features have been demonstrated to relate to changes in affective states. It may be observed that the EEG band-powers over the prefrontal cortex in a wide range of frequency bands and the band-powers in the parietal cortex in the alpha band are most often reported to relate to affective state changes.

Asymmetry features make use of a difference in the relative intensity of activity, typically, between the left and right hemispheres when experiencing different affective states. Specifically, when experiencing changing levels of valence or arousal the mean amplitude of EEG recorded over the right hemisphere differs significantly from the left hemisphere [63]. This effect is most pronounced in the pre-frontal cortex [55].

Network features are used to provide a measure of the amount of communication between different cortical regions $[64,65,66]$. This gives an indication of which cortical regions are either in direct communication with one another or are influenced by a common (but potentially unobserved) neural generator [67]. Changes in ongoing neural network activity have been observed to reflect changes in both valence and arousal and may be exploited for use in aBCIs [55].

ERPs provide a phase-locked measure of a change in amplitude of ongoing oscillatory activity in the EEG that relates to a time-locked event [68]. They may only be used with synchronous BCIs (BCIs that rely on a stimuli presentation at a specific time) [31]. Thus, they are most commonly used in conjunction with emotive images to improve classification accuracies in BCIs designed to allow selection between different choices [59, 61, 62].

An alternative approach to affective state detection is to use a hybrid approach which combines EEG based features with one or more other physiological features [26]. Examples of this approach include the use of ECG and EEG for improved classification accuracies [69] and the combination of EEG and NIRS to understand affective responses to speech [56].

\section{Affective BCIs}

\subsection{Categories of aBCI}

BCIs, and by extension aBCIs, may be categorized as being either passive BCIs or active BCIs.

Passive BCIs use some measure of the users neurological activity to affect 
Table 2: Use of EEG band-power features to identify an individuals affective state in different frequency bands and spatial regions of the cortex. For each region of the cortex and EEG frequency band the total number of participants involved in all studies providing evidence of an involvement of that cortical region is listed, along with references to the supporting studies.

\begin{tabular}{|c|c|c|c|c|c|c|c|c|c|}
\hline & & \multicolumn{8}{|c|}{ Regions } \\
\hline & & Front. & Cent. & Occip. & Temp. & Pari. & Left & Right & All \\
\hline \multirow{5}{*}{ 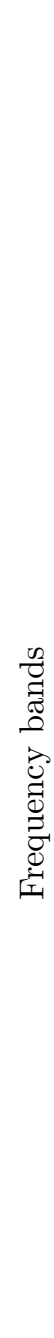 } & 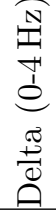 & $\begin{array}{c}93 \\
{[34,} \\
37,42, \\
16]\end{array}$ & $\begin{array}{c}20 \\
{[32} \\
34,42]\end{array}$ & $\begin{array}{c}79 \\
{[39,42]}\end{array}$ & & $\begin{array}{c}39 \\
{[34} \\
42,47]\end{array}$ & $\begin{array}{c}1 \\
{[32]}\end{array}$ & $\begin{array}{c}51 \\
{[47,} \\
16]\end{array}$ & $\begin{array}{c}63 \\
{[39]}\end{array}$ \\
\hline & 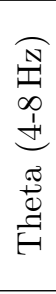 & $\begin{array}{c}241 \\
{[34,} \\
35,37, \\
38,39, \\
42,43, \\
44,47, \\
36]\end{array}$ & $\begin{array}{c}77 \\
{[34,} \\
35,38, \\
42,44]\end{array}$ & $\begin{array}{c}97 \\
{[39} \\
42,44]\end{array}$ & & $\begin{array}{c}81 \\
{[34,} \\
35,38, \\
42,43]\end{array}$ & & $\begin{array}{c}22 \\
{[43]}\end{array}$ & $\begin{array}{c}22 \\
{[48]}\end{array}$ \\
\hline & 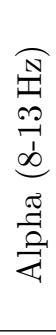 & $\begin{array}{c}329 \\
{[33,} \\
34,35, \\
36,37, \\
38,39, \\
41,42, \\
44,45, \\
47,36]\end{array}$ & $\begin{array}{c}141 \\
{[33,} \\
34,35, \\
38,39, \\
41,42, \\
44]\end{array}$ & $\begin{array}{c}35 \\
{[33} \\
42,44]\end{array}$ & $\begin{array}{c}63 \\
{[39]}\end{array}$ & $\begin{array}{c}153 \\
{[33,} \\
34,35, \\
38,41, \\
42,45]\end{array}$ & $\begin{array}{c}1 \\
{[33]}\end{array}$ & $\begin{array}{c}92 \\
{[33} \\
43,45]\end{array}$ & $\begin{array}{c}22 \\
{[48]}\end{array}$ \\
\hline & 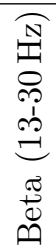 & $\begin{array}{c}218 \\
{[34,} \\
35,36, \\
37,39, \\
42,36, \\
16]\end{array}$ & $\begin{array}{c}77 \\
{[34,} \\
35,42, \\
46]\end{array}$ & $\begin{array}{c}16 \\
{[42]}\end{array}$ & $\begin{array}{c}63 \\
{[39]}\end{array}$ & $\begin{array}{c}112 \\
{[34,} \\
35,39, \\
42]\end{array}$ & & $\begin{array}{c}31 \\
{[16]}\end{array}$ & \\
\hline & 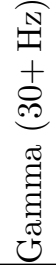 & $\begin{array}{c}213 \\
{[34,} \\
35,37, \\
39,40, \\
42,36, \\
16]\end{array}$ & $\begin{array}{c}140 \\
{[34,} \\
35,37, \\
39,42, \\
46]\end{array}$ & $\begin{array}{c}79 \\
{[39,} \\
42]\end{array}$ & $\begin{array}{c}74 \\
{[39,} \\
40]\end{array}$ & $\begin{array}{c}49 \\
{[34} \\
35,42]\end{array}$ & & $\begin{array}{c}94 \\
{[39,} \\
16]\end{array}$ & $\begin{array}{c}63 \\
{[39]}\end{array}$ \\
\hline
\end{tabular}


passive control over a system $[70,71,72]$. Thus, the BCI responds to the users current state without the user actually actively intending or willing the control to happen. An example of this may be a pBCI that uses a measure of the users current level of frustration to adjust the type of options presented to them [47]. A more frustrated user may be making lots of errors and may benefit from a reduced number of control options, which can lead to increased control accuracies.

By contrast, active BCIs allow the user to actively effect control over the system via their affective state. An example of this is the use of affective imagery to select between different control options [33]. For example, participants may be asked to imagine either low or high valence affective states in order to select between two control options.

\subsection{Use of affect in aBCI}

Affect may be employed in many different ways within both active and passive aBCIs. These different approaches may be grouped into the following categories.

1. Boosting classification performance.

2. Understanding the users emotions.

3. Modulating the users emotions.

Classification boosting refers to the use of affect to improve the classification accuracy of an active BCI that is intended for aiding a users ability to communicate or control their environment. For example, affect may be employed to identify incorrect selections (by identifying increases in user frustration). Alternatively, affect may be used directly to allow the user to select different options, for example affective imagery may be used to select between different choices made available to the user [33].

Understanding of user emotions can allow both active and passive aBCIs to respond better to a users needs [4]. For example, an understanding of a users current affective state can be used to modulate the contents of an aBCI controlled game [32].

Finally, a measurement of affect in an aBCI can be used to allow a passive aBCI to modulate the users emotions. This allows aBCI system to potentially be used for therapeutic purposes, where the aBCI is used to attempt to produce either short or long term improvements in an individuals emotions or moods [73]. An example of this approach is presented in the case study in section 6 .

There are several different potential applications of these various categories and types of aBCI. Specifically, aBCIs have been proposed for use in the following areas.

1. Therapy

2. Affective computing 


\section{Entertainment}

\section{Communication and control}

Therapeutic uses of BCI are diverse and range from rehabilitation of motor control after stroke through to treatment of attention deficit hyperactivity disorder. Chapter (?chapter link: Therapeutic applications of BCI technology?) provides an overview of the range of different therapeutic uses of BCI systems.

aBCIs have potential applications as aids and assistive tools in therapy. Specifically, by identifying and responding to an individuals current affective state an aBCI has the potential to be able to deliver stimuli to that user that is therapeutically beneficial. An example of this potential use in the case of a passive aBCI is discussed in section 6 .

Affective computing is the development of computer systems that can respond to a users affective state [74]. This allows a computer system to dynamically change its operating principles in order to meet the affective needs of a user. For example, an affective computing system may be used in e-learning applications in order to detect changes in a users affective state related to boredom (when the material is already known) or frustration (when the material is poorly understood) [75]. The system could then respond to these changes in affect by adapting the material presented to the student.

aBCIs have also been proposed for use in entertainment devices. For example, by identifying a users current affective state a passive aBCI is able to more effectively adapt an entertainment media (such as film, music, or stories) to better entertain the user. An example of this is described by Brouwer et al., who have developed an aBCI that responds to changes in user affect while they read passages from a novel [76]. The novel was written to contain multiple branching sections and different routes through the story were selected depending on the users changing affective state as the story progresses.

Finally, active aBCIs may also be used for communication and control. In this application type the user may attempt to actively modulate their affective state or engage in affective state imagery in order to select between different control options. Alternatively, affective states may be detected and used to inform the selection of control options via other means, such as ERP-based BCI.

\section{Case study: An affective BCMI}

We describe one example of an aBCI system developed for use as a passive BCI that uses a measure of a users current affective state in order to attempt to modulate their future affective state. Specifically, we describe an affective brain-computer music interface (aBCMI) developed in [69]. This aBCMI system is designed to detect a users current affective state and, given that affective state and a target affective state, use music generator in order to modulate the user's affective state. 


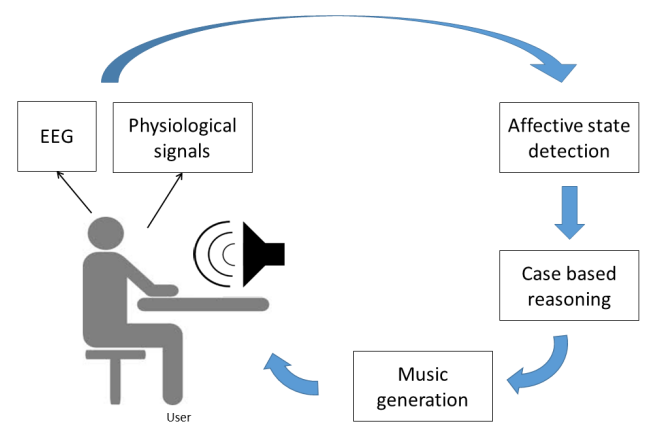

Figure 4: An affective brain-computer music interface (aBCMI).

The aBCMI provides a potential tool for use in music therapy by utilizing the feedback loop provided by the BCI system to allow modulation of a users affective state. BCMIs are an emerging form of BCI system designed to allow their users to interact with or create music. Chapter (?chapter link 29, BCI for music making?) provides an overview of the history and current state-of-the-art in the development of BCMI systems. The aBCMI system we describe here (illustrated in figure 4) includes four stages.

First, EEG and other physiological signals are recorded from the user and preprocessed to remove artefacts. Second, these preprocessed signals are used to identify the user's current affective state. The third step is then a case-based reasoning system, which is used to identify the specific modulations of the music played to the user in order to move them from their current affective state to a new target affective state. Finally, the fourth stage of the system is a music generator, which is used to generate music in order to attempt to move the user closer to the target affective state.

This aBCMI system was evaluated on a population of 20 healthy participants in order to determine whether it was able to successfully modulate their current affective states. Participants were asked to attend 5 separate sessions, the first four of which were used to train the aBCMI system and the fifth of which was used to evaluate the systems ability to achieve 4 key targets.

1. Make the user happier (increase the valence they report).

2. Make the user calmer (decrease arousal).

3. Reduce the users stress (increase valence and decrease arousal).

4. Excite the user (increase arousal).

The success of the system was evaluated by measuring participants selfreports of their current felt emotions, which were reported on a continuous basis using the FEELTRACE reporting tool [24]. Participant reports during 


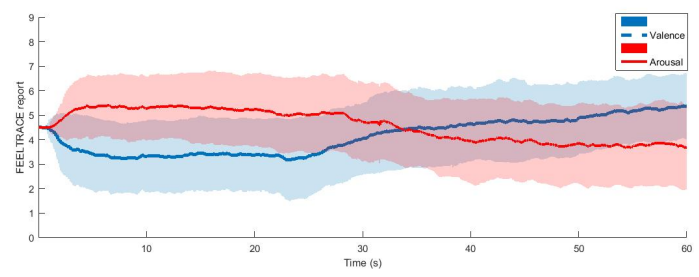

Figure 5: Mean FEELTRACE reports from all users of the aBCMI under the 'make happier' condition.

trials in which the aBCMI attempted to achieve each of the four goals were compared against the trajectory of the change in affective state that is intended during each of the key system goals. For example, in trials for which the goal of the aBCMI was to induce an increase in the users valence (make the user happier) the valence reported by the users was evaluated to determine whether it significantly increased over the course of the trial.

This is illustrated in figure 5, which shows the mean FEELTRACE reports from all aBCI users during the 'make happier' condition. Note that the report of valence increases over the course of the trial, a change that was observed to be statistically significant $(p<0.01)$.

The aBCMI was able to significantly increase the valence reported by its users during online evaluation. It was also able to significantly decrease users arousal and reduce the reported stress of its users (simultaneously decreasing arousal and increasing valence). However, it was unable to increase the arousal reported by its users, which may be due to the type of music generated by the aBCMI. Specifically, classic monophonic piano music was generated by the system and this genre of music may be less likely to induce excitement and, therefore, increases in arousal levels in listeners.

\section{Guide for developing aBCIs}

Based upon our observations from the literature a set of guidelines are provided to highlight key considerations when developing an aBCI.

First, identify the purpose of the aBCI. There are a wide range of different possible application areas for aBCI, such as aiding with communication or movement, therapy, or aiding with artistic expression, as well as new and previously unexplored areas. The first step in developing the aBCI should be to clearly identify the application area and the purpose of the aBCI.

Second, the category the aBCI falls into should be identified. aBCIs can be categorized as either active or passive (or a hybrid approach that combines both) and determining this category impacts significantly on how it is designed.

Third, identify how affect will be used within the aBCI. Measures of affect can be used in a wide range of different ways in aBCI, from aiding with 
communication / control accuracy, to providing a form of therapeutic feedback to the user. Additionally, affect may be the only control signal in your aBCI or it may be used in combination with other signals (such as ERPs).

Fourth, define how affect will be categorized and measured within the aBCI. Affect can be described within a range of different frameworks and measured via a variety of different metrics and it is important to identify which framework is to be used prior to constructing an aBCI.

Fifth, identify the affective state detection method to use in the aBCI and verify that it works correctly. This may involve offline studies, simulations, or pilot experiments, depending on the type of affective state detection method employed.

Sixth, it is important to consider inter-trial and inter-user variability in affective state responses. Affect is a fluid process that changes non-linearly over time and with respect to multiple factors. It also varies hugely between people. Thus, an affective stimuli may have very different effects on different users.

Seventh, it is also important to consider non-stationarity of affect. Affective state changes are not stationary over time and such non-stationarity needs to be considered when designing an aBCI. For example, in the case study described in section 6 the affective brain-computer music interface uses generated music to avoid the well-known effects of repeat exposure to the same stimuli on affective responses.

Eighth, it is important to remove artefacts from the neurological data used to control the aBCI. Artefacts are a problem for all BCI systems and need to be removed to ensure intention / affective state detection is accurate. However, they can be more of a problem for aBCIs where the most frequently used affective state detection methods are based on the pre-frontal asymmetry, a measure recorded from the EEG electrodes positioned closest to the eyes, and therefore the most susceptible to interruption by blink artefacts.

Finally, it is important to plan an appropriate testing strategy. Validating the efficacy of an aBCI can be more challenging than other types of BCI due to the inherent challenges in identifying a users 'ground truth', a measure of their actual affective state. Thus, experiments need careful designing in order to allow the aBCI to be rigorously evaluated.

\section{Summary}

Affective BCIs incorporate a measure of the user's affective state in order to provide a method for either improving BCI performance or widening the range of applications to which BCI can be applied. Specifically, aBCIs have been developed as tools for entertainment, aids to communication, and as therapeutic devices.

By including an understanding of affect an aBCI has the potential to respond more dynamically to a user's needs. This allows BCI applications to be expanded in scope and variety and, ultimately, has the potential to lead to improvements 
in the quality of life for a large number of people.

\section{References}

[1] J. R. Wolpaw, "Brain-computer interfaces as new brain output pathways." The Journal of physiology, vol. 579, no. Pt 3, pp. 613-619, mar 2007.

[2] T. O. Zander and S. Jatzev, "Detecting affective covert user states with passive brain-computer interfaces," in 2009 3rd International Conference on Affective Computing and Intelligent Interaction and Workshops. IEEE, sep 2009, pp. 1-9. [Online]. Available: http://ieeexplore.ieee.org/document/5349456/

[3] F. Nijboer, F. O. Morin, S. P. Carmien, R. A. Koene, E. Leon, and U. Hoffmann, "Affective brain-computer interfaces: Psychophysiological markers of emotion in healthy persons and in persons with amyotrophic lateral sclerosis," in 2009 3rd International Conference on Affective Computing and Intelligent Interaction and Workshops. IEEE, sep 2009, pp. 1-11. [Online]. Available: http://ieeexplore.ieee.org/document/5349479/

[4] C. Mühl, B. Allison, A. Nijholt, and G. Chanel, "A survey of affective brain computer interfaces: principles, state-of-the-art, and challenges," BrainComputer Interfaces, vol. 1, no. 2, pp. 66-84, apr 2014. [Online]. Available: http://www.tandfonline.com/doi/abs/10.1080/2326263X.2014.912881

[5] L. F. Barrett, B. Mesquita, K. N. Ochsner, and J. J. Gross, "The experience of emotion." Annual review of psychology, vol. 58, pp. 373-403, 2007. [Online]. Available: http://www.ncbi.nlm.nih.gov/pubmed/17002554 http://www.pubmedcentral.nih.gov/articlerender.fcgi?artid=PMC1934613

[6] J. A. Wiles and T. B. Cornwell, "A Review of Methods Utilized in Measuring Affect, Feelings, and Emotion in Advertising," Current Issues and Research in Advertising, vol. 13, no. 1-2, pp. 241-275, mar 1991. [Online]. Available: http://www.tandfonline.com/doi/abs/10.1080/01633392.1991.10504968

[7] J. A. Russell, "A circumplex model of affect." Journal of Personality and Social Psychology, vol. 39, no. 6, pp. 1161-1178, 1980.

[8] U. Schimmack and A. Grob, "Dimensional models of core affect: a quantitative comparison by means of structural equation modeling," European Journal of Personality, vol. 14, no. 4, p. 21, 2000.

[9] M. Zentner, D. Grandjean, and K. R. Scherer, "Emotions evoked by the sound of music: Characterization, classification, and measurement." Emotion, vol. 8, no. 4, pp. 494-521, 2008. [Online]. Available: http://doi.apa.org/getdoi.cfm?doi=10.1037/1528-3542.8.4.494 
[10] M. Pantic and L. Rothkrantz, "Toward an affect-sensitive multimodal human-computer interaction," Proceedings of the IEEE, vol. 91, no. 9, pp. 1370-1390, sep 2003. [Online]. Available: http://ieeexplore.ieee.org/document/1230215/

[11] J. F. Brosschot and J. F. Thayer, "Heart rate response is longer after negative emotions than after positive emotions," International Journal of Psychophysiology, vol. 50, no. 3, pp. 181-187, 2003.

[12] B. M. Appelhans and L. J. Luecken, "Heart rate variability as an index of regulated emotional responding." Review of General Psychology, vol. 10, no. 3, pp. 229-240, 2006. [Online]. Available: http://doi.apa.org/getdoi.cfm?doi=10.1037/1089-2680.10.3.229

[13] F. Agrafioti, D. Hatzinakos, and A. K. Anderson, "ECG Pattern Analysis for Emotion Detection," IEEE Transactions on Affective Computing, vol. 3, no. 1, pp. 102-115, jan 2012. [Online]. Available: http://ieeexplore.ieee.org/document/5999653/

[14] C. Landis and W. A. Hunt, "The conscious correlates of the galvanic skin response." Journal of Experimental Psychology, vol. 18, no. 5, pp. 505-529, 1935. [Online]. Available: http://content.apa.org/journals/xge/18/5/505

[15] M. V. Villarejo, B. G. Zapirain, and A. M. Zorrilla, "A stress sensor based on Galvanic Skin Response (GSR) controlled by ZigBee." Sensors (Basel, Switzerland), vol. 12, no. 5, pp. 6075-101, jan 2012. [Online]. Available: http://www.pubmedcentral.nih.gov/articlerender.fcgi?artid=3386730\&tool=pmcentrez\&rendertype=abst

[16] I. Daly, D. Williams, J. Hallowell, F. Hwang, A. Kirke, A. Malik, J. Weaver, E. Miranda, and S. J. Nasuto, "Music-induced emotions can be predicted from a combination of brain activity and acoustic features." Brain and cognition, vol. 101, pp. 1-11, dec 2015. [Online]. Available: http://www.sciencedirect.com/science/article/pii/S0278262615300142

[17] J. A. Etzel, E. L. Johnsen, J. Dickerson, D. Tranel, and R. Adolphs, "Cardiovascular and respiratory responses during musical mood induction." International journal of psychophysiology : official journal of the International Organization of Psychophysiology, vol. 61, no. 1, pp. 57-69, jul 2006. [Online]. Available: http://www.ncbi.nlm.nih.gov/pubmed/16460823

[18] C. M. Jones and T. Troen, "Biometric valence and arousal recognition," in Proceedings of the 2007 conference of the computer-human interaction special interest group (CHISIG) of Australia on Computer-human interaction: design: activities, artifacts and environments - OZCHI '0\%. New York, New York, USA: ACM Press, 2007, p. 191. [Online]. Available: http://portal.acm.org/citation.cfm?doid=1324892.1324929

[19] P. Ekman, "Facial expression and emotion." American Psychologist, vol. 48, no. 4, pp. 384-392, 1993. 
[20] A. Gabrielsson, "Emotion perceived and emotion felt: Same or different?" Musicae Scientiae, vol. Spec Issue, pp. 123-147, 2001.

[21] M. M. Bradley and P. J. Lang, "Measuring emotion: the Self-Assessment Manikin and the Semantic Differential." Journal of behavior therapy and experimental psychiatry, vol. 25, no. 1, pp. 49-59, mar 1994. [Online]. Available: http://www.ncbi.nlm.nih.gov/pubmed/7962581

[22] J. Morris, "Observations: SAM: The Self-Assessment Manikin; An Efficient Cross-Cultural Measurement of Emotional Response," Journal of Advertising Research, vol. 35, no. 8, 1995.

[23] J. K. Vuoskoski and T. Eerola, "Measuring music-induced emotion: A comparison of emotion models, personality biases, and intensity of experiences," Musicae Scientiae, vol. 15, no. 2, pp. 159-173, jul 2011. [Online]. Available: http://msx.sagepub.com/content/15/2/159.short

[24] R. Cowie, E. Douglas-Cowie, S. Savvidou, E. McMahon, M. Sawey, and M. Schröder, "FEELTRACE': An Instrument For Recording Perceived Emotion In Real Time," in Proceedings of the ISCA Workshop on Speech and Emotion, 2000, pp. 19-24. [Online]. Available: http://citeseerx.ist.psu.edu/viewdoc/summary?doi=10.1.1.58.7528

[25] R. Cowie, G. McKeown, E. Douglas-Cowie, G. Caridakis, K. Karpouzis, M. Wallace, L. Kessous, N. Amir, R. Cowie, R. Cornelius, R. Cowie, E. Douglas-Cowie, N. Tsapatsoulis, G. Votsis, S. Kollias, W. Fellenz, J. G. Taylor, E. Douglas-Cowie, N. Campbell, R. Cowie, P. Roach, L. F. Barrett, J. A. Russell, J. R. Fontaine, K. R. Scherer, E. B. Roesch, P. Ellsworth, J. Forgas, G. H. Bower, S. Ioannou, A. Raouzaiou, V. Tzouvaras, T. Mailis, K. Karpouzis, K. Kollias, C. Krumhansl, R. W. Levenson, J. M. Gottman, G. Luck, P. Toiviainen, J. Erkkila, O. Lartillot, K. Riikkila, A. Makela, J. Mayer, D. Caruso, P. Salovey, A. Mehrabian, D. S. Messinger, T. Cassel, S. Acosta, Z. Ambadar, J. F. Cohn, F. Nagel, R. Kopiez, O. Grewe, E. Altenmüller, R. Reisenzein, J. Russell, J. A. Russell, D. Sander, D. Grandjean, K. Scherer, H. Schlosberg, E. Schubert, E. Shriberg, A. Stolcke, D. HakkaniTur, G. Tur, I. Sneddon, G. McKeown, M. McRorie, T. Vukicevic, C. Stevens, E. Schubert, R. Morris, M. Frear, S. Stevens, and J. Sutton, "Tracing Emotion," International Journal of Synthetic Emotions, vol. 3, no. 1, pp. 1-17, jan 2012. [Online]. Available: http://services.igiglobal.com/resolvedoi/resolve.aspx?doi=10.4018/jse.2012010101

[26] G. Pfurtscheller, B. Z. Allison, C. Brunner, G. Bauernfeind, T. SolisEscalante, R. Scherer, T. O. Zander, G. Müller-Putz, C. Neuper, and N. Birbaumer, "The hybrid BCI," Frontiers in Neuroprosthetics, vol. 4, no. $30,2010$. 
[27] G. R. Müller-Putz, C. Breitwieser, F. Cincotti, R. Leeb, M. Schreuder, F. Leotta, and Et.al., "Tools for Brain-Computer Interaction: A General Concept for a Hybrid BCI." Front Neuroinform, vol. 5, p. 30, jan 2011.

[28] B.-K. Min, M. J. Marzelli, and S.-S. Yoo, "Neuroimaging-based approaches in the brain-computer interface." Trends in biotechnology, vol. 28, no. 11, pp. 552-60, nov 2010. [Online]. Available: http://dx.doi.org/10.1016/j.tibtech.2010.08.002

[29] B. Graimann, G. Pfurtscheller, B. Allison, and C. Neuper, Brain-Computer Interfaces, ser. The Frontiers Collection, B. Graimann, G. Pfurtscheller, and B. Allison, Eds. Berlin, Heidelberg: Springer, 2010. [Online]. Available: http://www.springerlink.com/content/k5x8743w21p8rx50/

[30] G. Liberati, R. Veit, S. Kim, N. Birbaumer, C. von Arnim, A. Jenner, D. Lule, A. C. Ludolph, A. Raffone, M. O. Belardinelli, J. D. da Rocha, and R. Sitaram, "Development of a Binary fMRI-BCI for Alzheimer Patients: A Semantic Conditioning Paradigm Using Affective Unconditioned Stimuli," in 2013 Humaine Association Conference on Affective Computing and Intelligent Interaction. IEEE, sep 2013, pp. 838-842. [Online]. Available: http://ieeexplore.ieee.org/document/6681549/

[31] G. R. Müller-Putz, R. Scherer, G. Pfurtscheller, and R. Rupp, "Brain-computer interfaces for control of neuroprostheses: from synchronous to asynchronous mode of operation." Biomed Tech (Berl), vol. 51, no. 2, pp. 57-63, jul 2006. [Online]. Available: $\quad$ http://www.degruyter.com/view/j/bmte.2006.51.issue2/bmt.2006.011/bmt.2006.011.xml http://www.ncbi.nlm.nih.gov/pubmed/16915766

[32] B. Reuderink, A. Nijholt, and M. Poel, "Affective Pacman: A Frustrating Game for Brain-Computer Interface Experiments." Springer Berlin Heidelberg, 2009, pp. 221-227. [Online]. Available: http://link.springer.com/10.1007/978-3-642-02315-6_23

[33] S. Makeig, G. Leslie, T. Mullen, D. Sarma, N. Bigdely-Shamlo, and C. Kothe, "First Demonstration of a Musical Emotion BCI," Affecti. Comput. and Int. Interact. Lect. Notes in Comp. Sci., vol. 6975, pp. 487-496, 2011.

[34] O. AlZoubi, R. A. Calvo, and R. H. Stevens, "Classification of EEG for Affect Recognition: An Adaptive Approach," in AI 2009: Advances in Artificial Intelligence. Springer Berlin Heidelberg, 2009, pp. 52-61. [Online]. Available: http://link.springer.com/10.1007/978-3-642-10439-8_6

[35] S. Koelstra and I. Patras, "Fusion of facial expressions and EEG for implicit affective tagging," Image and Vision Computing, vol. 31, no. 2, pp. 164174, 2013. 
[36] P. Petrantonakis and L. Hadjileontiadis, "Emotion Recognition From EEG Using Higher Order Crossings," IEEE Transactions on Information Technology in Biomedicine, vol. 14, no. 2, pp. 186-197, mar 2010. [Online]. Available: http://ieeexplore.ieee.org/document/5291724/

[37] M. Mauri, V. Magagnin, P. Cipresso, L. Mainardi, E. N. Brown, S. Cerutti, M. Villamira, and R. Barbieri, "Psychophysiological signals associated with affective states," in 2010 Annual International Conference of the IEEE Engineering in Medicine and Biology. IEEE, aug 2010, pp. 3563-3566. [Online]. Available: http://ieeexplore.ieee.org/document/5627465/

[38] R. Khosrowabadi, A. Wahab, K. K. Ang, and M. H. Baniasad, "Affective computation on EEG correlates of emotion from musical and vocal stimuli," in 2009 International Joint Conference on Neural Networks. IEEE, jun 2009, pp. 1590-1594. [Online]. Available: http://ieeexplore.ieee.org/lpdocs/epic03/wrapper.htm?arnumber $=5178748$

[39] M. Stikic, R. R. Johnson, V. Tan, and C. Berka, "EEG-based classification of positive and negative affective states," Brain-Computer Interfaces, vol. 1, no. 2, pp. 99-112, may 2014. [Online]. Available: http://www.tandfonline.com/doi/abs/10.1080/2326263X.2014.912883

[40] M. M. Müller, A. Keil, T. Gruber, and T. Elbert, "Processing of affective pictures modulates right-hemispheric gamma band EEG activity." Clinical neurophysiology : official journal of the International Federation of Clinical Neurophysiology, vol. 110, no. 11, pp. 1913-20, nov 1999. [Online]. Available: http://www.ncbi.nlm.nih.gov/pubmed/10576487

[41] C. Mühl, A.-M. Brouwer, N. van Wouwe, E. van den Broek, F. Nijboer, and D. Heylen, "Modality-specific Affective Responses and their Implications for Affective BCI," in Fifth International Brain-Computer Interface Conference. Verlag der Technischen Universität, 2011, pp. 22-24.

[42] M. Walpulski, "EEG representation of emotion evoking pictures," 2008.

[43] L. Aftanas, A. Varlamov, S. Pavlov, V. Makhnev, and N. Reva, "Event-related synchronization and desynchronization during affective processing: emergence of valence-related time-dependent hemispheric asymmetries in theta and upper alpha band." The International journal of neuroscience, vol. 110, no. 3-4, pp. 197-219, 2001. [Online]. Available: http://www.ncbi.nlm.nih.gov/pubmed/11912870

[44] C. M. Krause, V. Viemerö, A. Rosenqvist, L. Sillanmäki, and T. Aström, "Relative electroencephalographic desynchronization and synchronization in humans to emotional film content: an analysis of the 4-6, 6-8, 8-10 and 10-12 Hz frequency bands." Neuroscience letters, vol. 286, no. 1, pp. 9-12, may 2000. [Online]. Available: http://www.ncbi.nlm.nih.gov/pubmed/10822140 
[45] T. A. Dennis and B. Solomon, "Frontal EEG and emotion regulation: electrocortical activity in response to emotional film clips is associated with reduced mood induction and attention interference effects." Biological psychology, vol. 85, no. 3, pp. 456-64, dec 2010.

[46] M. Soleymani, S. Asghari-Esfeden, M. Pantic, and Y. Fu, "Continuous emotion detection using EEG signals and facial expressions," in 2014 IEEE International Conference on Multimedia and Expo (ICME). IEEE, jul 2014, pp. 1-6. [Online]. Available: http://ieeexplore.ieee.org/lpdocs/epic03/wrapper.htm?arnumber $=6890301$

[47] B. Reuderink, C. Mühl, and M. Poel, "Valence, arousal and dominance in the EEG during game play," International Journal of Autonomous and Adaptive Communications Systems, vol. 6, no. 1, dec 2012. [Online]. Available: http://www.inderscienceonline.com/doi/abs/10.1504/IJAACS.2013.050691

[48] L. Rogenmoser, N. Zollinger, S. Elmer, and L. Jäncke, "Independent component processes underlying emotions during natural music listening." Social cognitive and affective neuroscience, vol. 7, no. 37, pp. 1-12, apr 2016. [Online]. Available: http://www.ncbi.nlm.nih.gov/pubmed/27217116

[49] M. M. Cavazza, G. G. Aranyi, F. F. Charles, J. J. Porteous, S. W. S. Gilroy, G. G. Jackont, I. I. Klovatch, G. G. Raz, N. J. N. J. Keynan, A. A. Cohen, and T. T. Hendler, "Frontal Alpha Asymmetry Neurofeedback for Brain-Computer Interfaces," 2014.

[50] L. A. Schmidt and L. J. Trainor, "Frontal brain electrical activity (EEG) distinguishes valence and intensity of musical emotions," Cognition $\mathcal{E}$ Emotion, vol. 15, no. 4, pp. 487-500, jul 2001. [Online]. Available: http://dx.doi.org/10.1080/02699930126048

[51] T. Canli, J. E. Desmond, Z. Zhao, G. Glover, and J. D. Gabrieli, "Hemispheric asymmetry for emotional stimuli detected with fMRI." Neuroreport, vol. 9, no. 14, pp. 3233-9, oct 1998. [Online]. Available: http://www.ncbi.nlm.nih.gov/pubmed/9831457

[52] A. Kirke and E. Miranda, "Combing EEG Frontal Asymmetry Studies with Affective Algorithmic Composition and Expressive Performance Models," pp. 1-4, 2011.

[53] L. I. Aftanas, N. V. Reva, L. N. Savotina, and V. P. Makhnev, "Neurophysiological correlates of induced discrete emotions in humans: an individually oriented analysis." Neuroscience and behavioral physiology, vol. 36, no. 2, pp. 119-30, mar 2006. [Online]. Available: http://www.ncbi.nlm.nih.gov/pubmed/16380825

[54] J. T. Cacioppo, "Feelings and emotions: roles for electrophysiological markers." Biological psychology, vol. 67, no. 1-2, pp. 235-43, oct 2004. [Online]. Available: http://www.ncbi.nlm.nih.gov/pubmed/15130533 
[55] I. Daly, A. Malik, F. Hwang, E. Roesch, J. Weaver, A. Kirke, D. Williams, E. Miranda, and S. J. Nasuto, "Neural correlates of emotional responses to music: an EEG study," Neuroscience Letters, vol. 573, pp. 52-57, may 2014.

[56] T. M. Rutkowski, Q. Zhao, A. Cichocki, T. Tanaka, and D. P. Mandic, "Towards Affective BCI/BMI Paradigms Analysis of fEEG and fNIRS Brain Responses to Emotional Speech and Facial Videos," in Advances in Cognitive Neurodynamics (II). Dordrecht: Springer Netherlands, 2011, pp. 671-675. [Online]. Available: http://www.springerlink.com/index/10.1007/978-90-481-9695-1_100

[57] H. Bakardjian, T. Tanaka, and A. Cichocki, "Emotional faces boost up steady-state visual responses for braincomputer interface," NeuroReport, vol. 22, no. 3, pp. 121-125, feb 2011. [Online]. Available: http://content.wkhealth.com/linkback/openurl?sid=WKPTLP:landingpage\&an=00001756201102160-00005

[58] G. Chanel, J. J. Kierkels, M. Soleymani, and T. Pun, "Short-term emotion assessment in a recall paradigm," International Journal of HumanComputer Studies, vol. 67, no. 8, pp. 607-627, 2009.

[59] J. K. Olofsson, S. Nordin, H. Sequeira, and J. Polich, "Affective picture processing: An integrative review of ERP findings," Biological Psychology, vol. 77, no. 3, pp. 247-265, mar 2008. [Online]. Available: http://www.ncbi.nlm.nih.gov/pubmed/18164800 http://www.pubmedcentral.nih.gov/articlerender.fcgi?artid=PMC2443061 http://linkinghub.elsevier.com/retrieve/pii/S0301051107001913

[60] C. A. Frantzidis, C. Bratsas, C. L. Papadelis, E. Konstantinidis, C. Pappas, and P. D. Bamidis, "Toward emotion aware computing: an integrated approach using multichannel neurophysiological recordings and affective visual stimuli." IEEE transactions on information technology in biomedicine: a publication of the IEEE Engineering in Medicine and Biology Society, vol. 14, no. 3, pp. 589-97, may 2010. [Online]. Available: http://www.ncbi.nlm.nih.gov/pubmed/20172835

[61] K. E. Briggs and F. H. Martin, "Affective picture processing and motivational relevance: Arousal and valence effects on ERPs in an oddball task," International Journal of Psychophysiology, vol. 72, no. 3, pp. 299-306, 2009.

[62] H. T. Schupp, B. N. Cuthbert, M. M. Bradley, J. T. Cacioppo, T. Ito, and P. J. Lang, "Affective picture processing: the late positive potential is modulated by motivational relevance." Psychophysiology, vol. 37, no. 2, pp. 257-61, mar 2000. [Online]. Available: http://www.ncbi.nlm.nih.gov/pubmed/10731776 
[63] N. A. Fox, "If it's not left, it's right. Electroencephalograph asymmetry and the development of emotion." The American psychologist, vol. 46, no. 8, pp. 863-72, aug 1991. [Online]. Available: http://www.ncbi.nlm.nih.gov/pubmed/1928939

[64] F. Varela, J. P. Lachaux, E. Rodriguez, and J. Martinerie, "The brainweb: phase synchronization and large-scale integration." Nature reviews. Neuroscience, vol. 2, no. 4, pp. 229-39, apr 2001. [Online]. Available: http://dx.doi.org/10.1038/35067550

[65] J. P. Lachaux, E. Rodriguez, J. Martinerie, and F. J. Varela, "Measuring phase synchrony in brain signals." Human brain mapping, vol. 8, no. 4, pp. 194-208, jan 1999.

[66] I. Daly, S. J. Nasuto, and K. Warwick, "Brain computer interface control via functional connectivity dynamics," Pattern Recognition, vol. 45, no. 6, pp. 2123-2136, jun 2012. [Online]. Available: http://dx.doi.org/10.1016/j.patcog.2011.04.034

[67] J. P. Lachaux, E. Rodriguez, M. L. V. Quyen, A. Lutz, J. Martinerie, and F. J. Varela, "Studying single-trials of phase synchronous activity in the brain," International Journal of Bifurcation and Chaos, vol. 10, no. 10, pp. 2429-2439, 2000.

[68] T. C. Handy, Event-related Potentials: A Methods Handbook. MIT Press, 2005. [Online]. Available: http://books.google.com/books?hl=en\&lr=\&id=OQyZEfgEzRUC\&pgis=1

[69] I. Daly, D. Williams, A. Kirke, J. Weaver, A. Malik, F. Hwang, M. Wairagkar, E. Miranda, and S. Nasuto, "An Affective Brain-computer music Interface," in Proceedings of the 6th International Brain-Computer Interface Meeting, organized by the BCI Society, 2016.

[70] E. Cutrell and D. Tan, "BCI for passive input in HCI," in Proc. ACM CHI 2008 Conference on Human Factors in Computing Systems Workshop on Brain-Computer Interfaces for HCI and Games, 2007.

[71] T. O. Zander, C. Kothe, S. Jatzev, and M. Gaertner, "Enhancing Human-Computer Interaction with Input from Active and Passive Brain-Computer Interfaces," in Proc. 4th Int. BCI Workshop and Training Course. Springer London, 2010, pp. 181-199. [Online]. Available: http://link.springer.com/10.1007/978-1-84996-272-8_11

[72] T. O. Zander and C. Kothe, "Towards passive brain-computer interfaces: applying brain-computer interface technology to human-machine systems in general." Journal of neural engineering, vol. 8, no. 2, p. 025005, apr 2011. [Online]. Available: http://www.ncbi.nlm.nih.gov/pubmed/21436512 
[73] I. Daly, D. Williams, A. Kirke, J. Weaver, A. Malik, F. Hwang, E. Miranda, and S. Nasuto, "Affective Brain-Computer Music Interfacing," Journal of Neural Engineering, vol. accepted, 2015.

[74] S. D'Mello, A. Graesser, B. Schuller, and J.-C. Martin, Eds., Affective Computing and Intelligent Interaction, ser. Lecture Notes in Computer Science. Berlin, Heidelberg: Springer Berlin Heidelberg, 2011, vol. 6975. [Online]. Available: http://link.springer.com/10.1007/978-3-642-24571-8

[75] J. Tao and T. Tan, "Affective Computing: A Review." Springer Berlin Heidelberg, 2005, pp. 981-995. [Online]. Available: http://link.springer.com/10.1007/11573548_125

[76] A.-M. Brouwer, M. Hogervorst, B. Reuderink, Y. van der Werf, and J. van Erp, "Physiological signals distinguish between reading emotional and non-emotional sections in a novel," Brain-Computer Interfaces, vol. 2, no. 2-3, pp. 76-89, apr 2015. [Online]. Available: http://www.tandfonline.com/doi/full/10.1080/2326263X.2015.1100037 